\title{
O ADVENTO DA ANIMAÇÃO DIGITAL Da animação 2D tradicional ao 3D
}

Resumo: O advento das novas tecnologias e a incorporação de técnicas digitais no processo de criação de um filme animado contribuiu para uma alteração de paradigma no cinema de animação. Com base nas reflexões de Marshall McLuhan acerca dos meios de comunicação e da tecnologia enquanto extensões do Homem, o presente artigo propõe uma reflexão sobre essa mesma transformação. Nesse sentido, discorre-se sobre os significados associados ao termo animação à luz do atual contexto digital em que se insere e de uma breve contextualização histórica. São analisadas as principais diferenças entre a animação 2D tradicional e o 3D considerando os padrões estéticos e narrativos instaurados pelos estúdios Walt Disney e Pixar. Paralelamente, discutir-se-ão as implicações do advento do 3D na indústria da animação, refletindo-se acerca das possibilidades oferecidas pela introdução de ferramentas digitais na produção de um filme animado.

Palavras-chave: Cinema de animação, 3D, digital, Disney, Mcluhan, Pixar.

\section{THE ADVENT OF DIGITAL ANIMATION \\ From traditional 2D animation to $3 \mathrm{D}$}

\begin{abstract}
The development of new technologies and incorporation of digital techniques in the process of making an animated film contributed to a paradigm shift in animation cinema. Based on Marshall McLuhan's studies about the media and technology as extensions of the man's body, the present article proposes a reflexion concerning that transformation. Regarding this, we explore the definition of animation in light of the actual digital context in which it belongs, supported by a brief historical contextualization. We analyse the main differences between traditional 2D and 3D animation, considering the aesthetic and narrative elements of Walt Disney's Studios and Pixar Animation Studios. Simultaneously, we discuss the implications of the beginning and evolution of the 3D in the animation industry, contemplating the possibilities offered by the introduction of digital tools in the process of making an animated film.
\end{abstract}

Keywords: Animation cinema, 3D, digital, Disney, Mcluhan, Pixar.

\footnotetext{
${ }^{1}$ Mestranda em Ciências da Comunicação: Cinema e Televisão pela Universidade Nova de Lisboa (Portugal) (catarinagil.1994@gmail.com)
} 


\section{Introdução}

"Animation is everything that people have called animation in the different historical periods", asseverava Giannalberto Bendazzi. Se nos primórdios do cinema de animação e na era pré-digital a designação do termo animação se relacionava essencialmente com a ilusão ótica obtida através da gravação de imagens, fotograma a fotograma, conferindo uma sensação de movimento, a utilização de computadores para obter resultados similares de modo substancialmente mais rápido veio transformar esta perceção.

Partindo da premissa de Marshall McLuhan, ao compreender os meios de comunicação ou a tecnologia, como extensões do Homem, seja a nível psicológico, físico, social ou intelectual, poderia afirmar-se que a "explosão" do digital no universo cinematográfico capturou o trabalho tradicionalmente manual dos animadores para o circunscrever às novas tecnologias. 0 computador e todos os seus apetrechos, assim como os softwares para eles disponibilizados, conduziram o cinema de animação a dimensões cada vez mais complexas. Afirmava Charles Solomon numa antologia de textos sobre The Art of the Animated Image: "Filmmaking has grown so complicated and sophisticated in recente years that simple definitions of techniques may no longer be possible. It may be unreasonable to expect a single word to summarize such diverse methods of creating images on film." (Solomon, 1987, p.12)

Efetivamente, o termo animar, assim como as palavras que deste derivam - animação, animado ou animador provêm do latim animare que significa "dar vida a" (Solomon, 1987, p.10) Contudo, esta designação parece insuficiente se considerados os múltiplos contextos nos quais a animação se insere atualmente, quer digam respeito ao contexto social, político, económico, estético ou provenientes do próprio imaginário do animador. Segundo a definição do animador Norman McLaren, citada por Solomon:

Animation is not the art of drawings that move, but rather the art of movements that are drawn. What happens between each frame is more important than what happens on each frame. The basic substance of the cinema is movement - at its lowest physical level, the movement of lightwaves and soundwaves... it is the motion that speak to us.

De acordo com McLaren, a essência da animação reside na expressividade conseguida através da dinâmica entre fotogramas. Animação é a criação de vida onde esta, numa primeira instância, não existe. É emoção. É o sentimento oferecido pelo animador à sua criação $\mathrm{e}$, posteriormente, ao espectador. Em contexto contemporâneo, porém, no pleno fervilhar de uma era digital, quando se alude ao termo animação fala-se numa multiplicidade de criações, desde filmes de animação, efeitos especiais, motion graphics, jogos para o telemóvel, computador ou internet, entre outras. Já Sébastien Denis declarava: "Definir a animação continua a ser, pela própria disparidade das técnicas e das filosofias pessoais do movimento e da vida que ela engendra, uma tarefa difícil, e até impossível" (Denis, 2010, p.13), citando logo de seguida Dick Tomasovick, o qual assevera que um dos traços identitários da animação é, efectivamente, a dificuldade em se atribuir uma identidade. Contudo, talvez o verdadeiro desafio já não passe por definir animação. Citando o animador Philip Kelly Denslow: "With the future digitalization of all media, all forms of production will perhaps be as much animation as anything else" (Pilling, 1997, p.4). O verdadeiro desafio talvez passe por compreender de que modo a utilização de ferramentas digitais no processo criativo e a deflagração do 3D no meio cinematográfico vieram alterar a relação do animador com a sua criação e a do espectador com o cinema de animação.

\section{Migração do papel para o computador}

O desenvolvimento da computação gráfica começou a despontar na década de 1960, no âmbito da aeronáutica para aplicação em simuladores de voo (Goodman, 1987, p.102) e outros aparelhos tecnológicos destinados ao treinamento militar ou ao progresso científico. Todavia, é somente a partir dos anos 1970 que se desenvolvem softwares capacitados a auxiliar o trabalho dos animadores, indo de encontro à sua curiosidade em introduzir técnicas inovadoras nos filmes de animação. Das experiências com o 
digital poderão destacar-se a conjugação entre a filmagem real e o desenho animado - The Yellow Submarine (1968) ou Who Framed Roger Rabbit (1988) - ou os efeitos especiais, salientando-se nesta área os realizadores George Lucas e Steven Spielberg com Star Wars (1977) ou E.T. (1989), respetivamente. Afirmava Solomon:

As interest in the technical aspects of film production grew more widespread during the '70's, viewers became aware that "animation" actually described a wide array of frame-by-frame filmmaking techniques, ranging from stop motion to drawing directly on film to photokinestasis. At the same time, sophisticated new processes involving special effects, computer graphics, motion control and video editing made it increasingly difficult to determine the boundaries between live action film making and animation.

De acordo com Solomon, através da introdução e aperfeiçoamento de técnicas digitais a animação passou a representar um vasto leque de processos digitais e a fronteira entre o cinema de animação e o cinema de imagem real tornou-se cada vez mais ténue. Terá sido Tron (1982) de Steven Lisberger, segundo Denis, o pioneiro na introdução da imagem de síntese (1) num filme de imagem real, mas é o sucesso das produções do estúdio Pixar, nomeadamente Luxo Junior (1986) e Tin Toy (1988), realizados por John Lasseter, a fomentar o mercado do 3D.

Pixar, fundada em 1979 como um segmento da Lucasfilm, a Graphics Group, torna-se uma companhia independente em 1986 quando é comprada por Steve Jobs. Em 1995, o sucesso de Toy Story marcaria o declínio comercial da animação 2D tradicional. Ainda hoje a imagem de síntese na animação "invade" as salas de cinema com um êxito fenomenal entre os espectadores. Dizia Sébastien Denis: "As técnicas digitais alteraram consideravelmente a relação dos espectadores com ela, e mudaram a própria produção a nível internacional. A imagem de síntese (o 3D) tornou-se, desde há alguns anos, dominante, sinal aparente do fim das técnicas mais artesanais.” (Denis, 2010, p.9)
Efetivamente, as novas tecnologias resultaram num impacto para diversas formas de arte e vieram desafiar uma vez mais a tentativa de definição do termo animação. A incorporação de ferramentas digitais no processo de criação de uma obra e a tão gradual quanto repentina passagem do papel, da pauta musical ou da tela de pintura para o computador vieram transformar o cinema de animação e outras formas de expressão artística. Com base nessa transmutação, Roger F. Malina, num artigo sobre a imagem e o cinema digitais, desdobra a sua atenção, numa primeira instância, sobre o impacto da utilização do computador em formas de arte já existentes como a música ou o cinema, afirmando:

One of the problems facing the artist using computers in pre-existing or traditional art forms is that the computer was not developed with the specific needs of artists in mind. The computer keyboard, mousse, digitizing tablet are all inferior tools for drawing compared to a piece of charcoal.

Segundo Malina, as ferramentas digitais poderão limitar a visão e emoção do artista ao condensarem em si mesmas uma série de propriedades intrínsecas a técnicas primitivamente artesanais como o desenho ou o domínio de um instrumento musical. $\mathrm{O}$ rato do computador ou a pen dos tablets adquirem a função de lápis, o monitor é uma nova janela de criação, adquirindo um estatuto similar ao da folha de papel ou da tela de pintura, e $o$ teclado do computador metamorfoseia-se no teclado de um piano ou nas cordas de uma guitarra. Aliás, Malina observa nos artistas utilizadores de ferramentas digitais a capacidade de criação de trabalhos "that either is indistinguishable $\square$ from that made using painting techniques or is equally successful artistically and aesthetically" (Malina, 1990, p.34). O computador, aliado a uma panóplia de software, habilita qualquer artista ou utilizador a produzir obras de génese especificamente artesanal através de fiéis reproduções digitais das técnicas tradicionais que lhes estão associadas. A criação de um desenho, por exemplo, é afastada de traços comuns à sua essência. $\mathrm{O}$ trabalho de um animador, usualmente assente na momentaneidade do traço, edificando, dese- 
nho a desenho, uma cena animada, pode agora ser complementado ou totalmente gerado segundo um processo de construção e moldagem digitais. Erros são facilmente apagados, cores agilmente alteradas, jogos de luzes e sombras eficazmente enquadrados...

De facto, o computador alcança de forma substancialmente mais rápida - e barata - o que tradicionalmente seria feito por um animador num estúdio de animação. De acordo com James F. Blinn, se determinada cena não corresponde ao desejado, bastaria inserir os dados no computador para que este produzisse as alterações pretendidas (Solomon, 1987, p.89). As relações passam a ser mediadas entre o Homem e a máquina, ao invés de permanecerem ao nível da interação social, de humano para humano. Existe no digital uma projeção de capacidades humanas. Dizia McLuhan: "all technologies are extensions of our physical and nervous systems" (McLuhan, 1964, p.90) e "Any extension, whether of skin, hand, or foot, affects the whole psychic and social complex" (McLuhan, 1964, p.4). O rato do computador passa a ser uma extensão da mão, o monitor uma extensão dos olhos, o computador uma extensão do cérebro e a tecnologia, em geral, torna-se uma extensão do Homem. "A tecnologia digital e a criação de softwares especializados contribuíram para que os artistas pudessem avançar em direção à almejada automatização do processo produtivo" (Ribeiro da Cruz, 2006, p.43).

Não obstante, de acordo com James F. Blinn, apesar das técnicas digitais permitirem alcançar o mesmo objetivo mais rapidamente do que se obteria convencionalmente, através de lápis e papel, "the instructions given to the computer must be more detailed and precise than those given to human assistants" (Solomon, 1987, p.89). Embora já se desenvolvam investigações e testes no sentido de elevar determinados softwares à simulação da personalidade de uma personagem, gerando automaticamente as suas reações perante diversas situações e aproximando o trabalho do animador ao de um realizador de cinema em filmagem real, o computador ainda é só e apenas uma máquina. É, portanto, mecânico. Na atual conjuntura, o computador funciona meramente como extensão do animador e não como cerne do âmago criativo.

\section{0 advento, triunfo e propagação do 3D}

Anteriormente ao êxito do 3D no meio cinematográfico, o 2D tradicional manteve-se durante um largo período de tempo característico do mercado da animação. A posterior ascensão da animação digital terá oferecido mudanças na relação do animador com a sua criação, na medida em que o trabalho por si desempenhado passou a ser mediado por programas de computador, não dependendo inteiramente das suas capacidades enquanto animador.

Por outro lado, o digital também transformou a relação do espectador com o cinema de animação, pois apesar da estrutura narrativa dos filmes ser praticamente idêntica, a animação 2D tradicional e 3D diferem nas estratégias utilizadas para o emprego desses mesmos modelos narrativos. Enquanto filmes de animação tradicionais como A Branca de Neve e os Setes Anões (Walt Disney, 1937), Mr. Bug Goes to Town (Fleisher Studios, 1941) ou La rosa di Bagdad (Ima-Film Productions, 1949), apelam a uma abordagem dramática, aliviada pela comicidade de personagens secundárias, nos filmes em 3D, tais como Toy Story (Pixar, 1995), Shrek (Dreamworks, 2001) ou Ice Age (Blue Sky Studios, 2002), não só é instaurado um novo paradigma estético apelando constantemente ao gag (2) visual e narrativo, como se alude a preocupações específicas da sociedade contemporânea. Nota-se uma diferença significativa entre a estética contemplativa dos filmes 2D, num apelo constante à emoção do espectador, contrariamente ao cinema de animação 3D, baseado fundamentalmente em estratégias de humor e reforçando "uma representação burlesca da realidade." (Ribeiro da Cruz, 2006, p.112)

Terá sido o estúdio Pixar um dos maiores impulsionadores do sucesso do mercado 3D justamente por a sua primeira longa-metragem de animação em 3D, Toy Story, ter quebrado com a opção estética, narrativa e dramática tradicional de Walt Disney. Apesar de os dois estúdios trabalharem representações de mundos imaginários numa base antropomórfica, nas produções de Walt Disney o real e imaginário geralmente fundem-se, enquanto na Pixar os dois mundos convivem num misto de comicidade e ironia. "A própria organização do mundo imaginário é criada à semelhança dos humanos: os bonecos de Toy 
Story se reúnem em assembleia; Nemo é um peixe que quer ir à escola; Sulley é um monstro cuja rotina inclui ir ao trabalho" (Ribeiro da Cruz, 2006, p.85) ao passo que nos filmes de Disney Dumbo é um elefante de circo; Bambi é um veado que vive na floresta; os 101 dálmatas são animais de estimação... É, para além disso, evidente a predominância de personagens antropomórficas nos filmes de animação 3D - note-se Finding Nemo (Pixar, 2002), Kung Fu Panda (Dreamworks, 2008), Rio (Blue Sky Studios, 2011) ou Zootopia (Disney, 2016) - onde o espectador consegue reconhecer características especificamente humanas em elementos da natureza, objetos ou animais. "Mainstream computer-animated films have ultimately been subsumed into a broader creative anthropomorphosis" (Holliday, 2016, p.247). O recente percurso da animação digital está, de facto, fortemente interligada com tais reproduções antropomórficas da realidade comandadas pelo gag.

Finalmente, se questionarmos qual a base do recente e contínuo sucesso dos filmes de animação produzidos em CGI, Sébastien Denis responde que a chave do êxito entre o público adulto poderá relacionar-se, efetivamente, com o uso recorrente de humor sofisticado ou com duplo sentido, afastando-se dos padrões estéticos e narrativos de Walt Disney, considerados infantilizantes. Relativamente aos mais jovens, Denis acredita poder dever-se, entre outros fatores, à semelhança entre a estética dos filmes de animação 3D com os jogos de vídeo 'Denis, 2010: 193-194). Aliás, num artigo sobre a convergência entre o universo dos jogos de vídeo e o cinema, Keith B Wagner e In-gyoo Jang utilizam como exemplo Wall-E (2008) da Pixar e Wreck it Ralph (2012) da Walt Disney, analisando a evolução da animação no modo como se foi interconectando com outros meios, nomeadamente com o âmbito dos jogos de vídeo. Afirmam: "What cinema and gaming now find in common with 3-D computer animation, amongst other things, is a platform for cultural expression that now overlaps and enmeshes itself in all three forms of visual media" (Wagner e Jang, 2016, p.131).

O êxito da animação 3D faz-se acompanhar por uma lógica económica que extrapola o seu suporte, daí o tremendo sucesso de Walt Disney, "incontestavelmente fi- gura de génio do marketing" (Denis, 2010, p.198). É num universo dinâmico, em constante comunicação com outros meios artísticos e culturais, que o cinema de animação adquire novas dimensões. Já não são apenas filmes, são um mercado mundial capturado pelo potencial das novas tecnologias.

\section{Possibilidades oferecidas pelas novas tecnologias}

Embora o cinema de animação 3D tenha atingido um tremendo sucesso, colocando o 2D tradicional numa dimensão nostálgica, ainda acordada nas recordações de infância de alguns espectadores, a animação tradicional prevalece, sendo, em diversos casos, complementada com tratamento digital. Cartoon Saloon, um estúdio irlandês, nomeado para um Óscar da Academia com o filme Song of the Sea (2014), é um exemplo da intercalação entre técnicas tradicionais com programas de edição digitais. Thomas Moore, um dos fundadores do estúdio, em entrevista sobre Song of the Sea dizia: "We used a hybrid of new and old techniques to create a traditional look, as I believe traditional 2D animation has a timeless appeal that can be watched even 20 years from now without seeming dated".

Também o animador George Griffin referia de que forma os avanços tecnológicos representavam uma promessa aos animadores, esclarecendo: "cheap software (...), intuitive graphic tablets, digital delivery systems for a variety of sites - all converge to enhance production and presentation" (Furniss, 2012, p.191) Conclusões, já analisadas por Philip Kelly Denslow num artigo sobre como as novas tecnologias, permitiram aos artistas ter acesso a uma panóplia de ferramentas digitais a um preço acessível e utilizá-las em prol das suas necessidades criativas:

Technology does hold out hope for independent artists to gain access to sophisticated tools as computers and digital reproduction become more and more economical. Visions of small-scale investment leading to large-scale access to markets using telecommunication networks, the aesthetic possibilities of replication and manipulation of existing or created material in the digital realm, and the popular- 
isation of an animator's personal vision are all parts of an optimistic scenario. Although digital imagery is leading us to a preoccupation with the realistic representation of ideas, at the same time these images are less fixed and more malleable than ever before.

Apesar da era digital ter brindado o cinema de animação com um mar de possibilidades até então nunca experienciadas, Denslow questiona-se até que ponto uma criação continuará a pertencer ao animador se grande parte dela for definida por um computador. Nesse sentido, pondera ainda se os criadores dos softwares não teriam parte do crédito naquela animação. A facilidade de partilha e distribuição na internet, igualmente evidenciada por Griffin, institui inevitavelmente uma rede de conexões entre animador, programador, realizador, utilizador e afins. Estabelece-se assim um paralelismo com o conceito de Aldeia Global, desenvolvido por McLuhan, com o intuito de explicar como as novas tecnologias e o fervilhar da era digital reduzem o planeta ao estatuto de aldeia, onde o mundo, de alguma forma, estaria interligado. Atualmente este conceito é facilmente aplicável tendo em consideração o grandioso fluxo de informação estimulado pela internet, a facilidade de comunicação com qualquer pessoa em qualquer parte do mundo, a rapidez de deslocação e, recuperando as reflexões de Roger F. Malina, os próprios softwares disponibilizados para o computador que colocam qualquer um em contacto com o cinema, a fotografia, a pintura, a música... No atual contexto contemporâneo, bastaria ter acesso a um computador e à internet para se realizar um filme de animação.

Na composição do seu último livro, The Medium is the Massage (1967), construído em parceria com o ilustrador Quentin Fiore, McLuhan debruça-se exatamente sobre os efeitos das novas tecnologias e dos meios de comunicação no Homem. Através de uma compilação de textos e imagens dispostos caoticamente ao longo de 160 páginas, o filósofo ilustra o modo como o desenvolvimento da tecnologia molda a sociedade e a sua perceção sobre o mundo. A composição dos diferentes elementos apresenta-se ao estilo de uma banda-desenhada que, embora fragmentada, é, tal qual um Little Nemo in
Slumberland (1905), muito rítmica e cinematográfica. No título, a subtil substituição da letra "e" em message pelo "a", oferece pistas sobre o efeito alienatório dos media e da tecnologia no Homem. McLuhan provoca o leitor, fá-lo questionar-se, auxiliando-se de uma construção imagética e textual que pode associar-se à ágil construção de sequências fílmicas animadas através de um computador e ao ritmo frenético a que a informação circula atualmente na dita Aldeia Global.

Numa diferente abordagem, esta igualmente evidenciada por Malina, o computador poderá ser encarado como um auxiliar na concretização de ideias até então nunca alcançadas devido à limitação tecnológica. A maleabilidade das ferramentas digitais oferece a qualquer aspirante a possibilidade de realizar um filme de animação, algo impensável no início do século XX. "A possibilidade de fazer mais no mundo virtual tornará mais eficientes os mecanismos do nosso mundo físico." (Schmidt e Cohen, 2013, p.24) Embora seja mais fácil desenhar o primeiro fotograma de uma animação em papel, o computador facilita o processo que daí advém e, portanto, aludindo às reflexões de James $\mathrm{F}$. Blinn, o seu verdadeiro encanto comparativamente às técnicas de animação convencionais está precisamente no facto de este acompanhar o animador, passo a passo, em direção a situações cada vez mais complexas e cativantes (Solomon, 1987, p.92) o que, gradualmente, alterará a forma de pensar do Homem.

\section{Uma nova forma de pensar}

Presentemente, o pensamento humano é dominado pela linguagem. Aliás, na teoria de Marshall McLuhan, a linguagem é um meio (ou uma tecnologia) por ser uma extensão da consciência humana. Ela representa ideias, pensamentos e emoções. McLuhan confere, de facto, grande importância ao desenvolvimento da linguagem humana quando escreve: "It is the extension of man in speech that enables the intellect to detach itself from the vastly wider reality. Without language... human intelligence would have remained totally involved in the objects of its attention." (McLuhan, 1964, p.79) Para o filósofo, a linguagem foi a chave para a evolução de extensões tecnológicas como a escrita, por exemplo, a qual 
representou uma evolução na tecnologia da linguagem e alterou o modo de pensamento até então vigente. A escrita auxiliou ao desenvolvimento e cimentação da memória, tornando-se uma extensão desta e do discurso.

Transpondo o olhar de McLuhan para o universo da animação digital, compreendendo a tecnologia como uma extensão das nossas faculdades biológicas e do computador como uma extensão do nosso cérebro, dizia James F. Blinn:

Consider making outlines for a paper and rearranging the topics as new ones come to mind. Now consider the same thinking process applied to pictures. Often people draw doodles or sketches to help them think. The technology of fast computer animation will enable thinkers to re-arrange and reformat their thoughts with the same ease. It is ease of use and quick feedback that makes this process useful. Computers are now just reaching this threshold of usefulness.

De acordo com as reflexões de Blinn, o computador poderá, tal como a escrita, reformatar o modo de pensar do Homem à medida que é desenvolvido e experienciado o seu potencial técnico e criativo. Embora os filmes de animação 3D sigam no caminho da estagnação, ao estarem dominadas pelo mercado, a sua qualidade técnica é indubitavelmente aprimorada a cada nova produção. $\mathrm{O}$ cinema de animação continuará o seu processo de transformação ao ritmo das exigências da era digital, transportada pelo crescente desenvolvimento tecnológico. Se hoje Walt Disney é relembrado pelo sucesso da animação 2D tradicional na década de 1930, Pixar ficará na história como responsável pelo triunfo do 3D no novo milénio. Inevitavelmente, o Homem verá a sua linguagem sofrer um novo processo de evolução social, cultural e histórico, da mesma forma que sucedeu com a invenção da escrita e com o progresso da eletricidade.

\section{Considerações finais}

$\mathrm{O}$ leque de possibilidades disponibilizado pelo crescente desenvolvimento tecnológico tornou-se imenso, repercutindo-se nos mais variados sectores. $\mathrm{O}$ cinema de animação que "é, antes de mais, cinema" (Denis, 2010, p. 7), foi uma das áreas atingidas pelo brotar da pósmodernidade, expandido as suas potencialidades criativas a uma dimensão que atualmente extrapola o meio cinematográfico.

As novas tecnologias proporcionaram um crescimento exponencial no universo da animação a nível estético, narrativo e social, maturando-a enquanto uma técnica cinematográfica cada vez mais complexa e absorvida nas suas idiossincrasias. Assim como a década de 1930 retratou o auge das produções Disney, embebidas numa completação visual estética e narrativamente dramática, o cinema de animação vive outro momento de glória com o sucesso do 3D, impulsionado pela irreverência de Pixar, a qual se ergueu em força em produções inovadoras e completamente digitais. "In the contemporary era, animation both constitutes a particular, and a distinctive form of cinema and operates as a language of expression in a range of diverse forms" (Nelmes, 2012, p.230).

Animação talvez já não seja passível de uma definição comum no meio cinematográfico, mas aporta agora em si uma panóplia de variações em diversos âmbitos - cinema experimental, arte contemporânea, jogos de vídeo, televisão... - que a enriquecem como técnica e a valorizam enquanto campo de investigação. Retomando a citação inicial de Giannalberto Bendazzi, "Animation is everything that people have called animation in the different historical periods".

\section{NOTAS:}

(1) - Imagens de síntese são imagens em 3 dimensões geradas por computador, vulgarmente designadas por CGI (Computer-Generated Image).

(2) - Gag é um termo inglês que se refere a piada, efeito cómico. 


\section{Referências bibliográficas}

Bendazzi, G. (2004). Defining Animation - A proposal. Disponível em: http://giannalbertobendazzi.com/wp-content/ uploads/2013/08/Defining_Animation-Giannalberto_Bendazzi 2004.pdf.

Denis, S. (2010). $O$ cinema de animação. Lisboa: Edições Texto \& Grafia.

Furniss, M. (2012). Animation: Art and industry. Leicester: John Libbey Publishing Ltd.

Goodman, C. (1987). Digital visions: Computers and art. New York: Harry N. Abrams, Inc.

Holliday, C. (2016). 'I'm not a real boy, I'm a puppet': Computer-animated films and anthropomorphic subjectivity. In Animation: an interdisciplinary journal, 11(3), 246-262. doi:10.1177/1746847716661456.

Malina, R. (1990). Digital image: Digital cinema: The work of art in the age of post-mechanical reproduction. In Leonardo. Supplemental Issue, 3, 33-38. Disponível em: http://www.jstor.org/stable/1557892.

Mcluhan, M.; Quentin, F. (1967). The medium is the massage: An inventory of effects. London: Penguin Books.

Mcluhan, M. (1994). Understanding media: The extensions of man. Cambridge: The MIT Press. (Orig. Ed.: 1964).

Nelmes, J. (2012). Introduction to film studies: Fifth Edition. London \& New York: Routledge.

Pilling, J. (1997). A reader in animation studies. London: John Libbey \& Co. Pty Ltd.

Ribeiro da Cruz, P. (2006). Do Desenho Animado à Computação Gráfica: A Estética da Animação à Luz das Novas Tecnologias. Salvador: Universidade Federal da Bahia. Disponível em: http://www.bocc.ubi.pt/pag/cruz-pauladesenho-animado-computacao-grafica.pdf.

Schmidt, E.; Cohen, J. (2013). A nova era digital. Lisboa: Dom Quixote.

Solomon, C. (1987). The art of the animated image: An Anthology. Los Angeles: The American Film Institute.

Wagner, K. B.; Jang, I. (2016). The 3-D Animated codescape: Imperfection and digital labor zones in Wall-E (2008) and Wreck-It Ralph (2012). In Animation: An Interdisciplinary Journal, 11(2), 130-145. doi:10.1177/1746847716638267. 